\title{
Identification and observation in the anode line of PEM fuel cell stacks
}

\author{
C. Kunusch, Member, IEEE, J.A. Moreno, Member, IEEE and M.T. Angulo
}

\begin{abstract}
In this work, some potential identification/observation problems that arise in the anode line of a typical Polymer Electrolyte Membrane (PEM) fuel cell based system are highlighted and then solved. They involve estimating the hydrogen input flow at the stack anode and the water transport across the membrane. It is argued that estimating the membrane water transport is of special interest since it gives relevant information on the actual performance and state of the fuel cell. Both problems are solved by constructing robust observers and parameter identification algorithms based on the Generalized Super-Twisting Algorithm.

Index Terms-Fuel cell; observer; estimation; sliding-modes.
\end{abstract}

\section{INTRODUCTION}

Fuel cells are complex dynamic systems usually described by nonlinear uncertain models. In order to reduce the number of sensors and estimate inaccessible variables, increasing research activities have been performed addressing observation problems in fuel cells.

In [2], the authors design observers for estimating the hydrogen partial pressure based on the stack output voltage. However, the proposed strategy relies on the internal model of the fuel cell voltage, which is usually unknown. Görgün et al. have also presented an estimation algorithm for the membrane water content in a polymer electrolyte membrane, but is also based on the voltage internal model [3]. Pukrushpan et al. have proposed an approach to partially estimate the state of a fuel cell based system using a Kalman filter that considers a linearized model [4]. McKay and Stefanopoulou have employed open loop nonlinear observers based on lumped dynamic models for estimating the anode and cathode relative humidities [1]. This last reference also presents experimental results, which is a major breakthrough in PEM fuel cells water content estimation.

In this work, we present another approach to the anode line estimation problem of water transport in polymeric membranes. Based on the validated lumped parameter model presented in [5], an estimation of the membrane water transport is obtained. The idea is neither using the internal model of the membrane nor the model of the stack voltage, because this may lead to a lack of robustness due to the complex and unknown time-variant models. The presented approach

C. Kunusch is with Institut de Robòtica i Informàtica Industrial (CSIC-UPC), Llorens i Artigas, 4-6, 08028 Barcelona, Spain (e-mail: ckunusch@iri.upc.edu).

J.A. Moreno is with the Instituto de Ingeniería, Universidad Nacional Autónoma de México (UNAM), Coyoacán. 04510, México (e-mail: jmorenop@ii.unam.mx).

M.T. Angulo is with División de Posgrado e Investigación, Facultad de Ingeniería, Universidad Autónoma de Querétaro (FI-UAQ), México (e-mail: darkbyte@gmail.com). uses the system's structure (but not its uncertain parameters) and three standard available measurements. Based on the Generalized Super-Twisting (GST) algorithm, the proposal robustly solves the estimation problem in finite time. The contribution is relevant and novel in terms of potential implementation in actual systems and due the inherent robustness and convergence properties of the GST algorithm.

In the following, an experimentally validated analytical model of a laboratory Polymer Electrolyte Membrane Fuel Cell (PEMFC) is presented. This model was specially developed for nonlinear control and observation issues. The modelling process is conducted following a modular methodology combining a theoretical approach, together with an empirical analysis based on experimental data. The proposed semi-empirical model is capable to adequately describe the interaction between the different subsystems, while retaining parameters and variables that have physical significance. The systematic procedure developed in [5] and [6], is presented in a way such that it can be used as a general modelling guideline, being straightforward to adapt to different fuel cell systems with few modifications. Concisely, the actual laboratory test plant under consideration mainly comprises a central PEMFC stack and ancillary units: air compressor, hydrogen storage tank, gases humidifiers and line heaters (see schematic representation in Fig. 1). In addition, to measure the required experimental data, a particular sensor array was incorporated into the system.

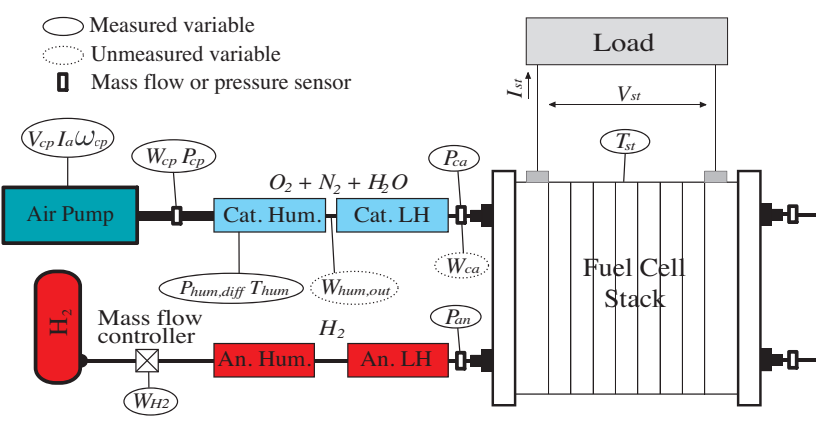

Fig. 1. Schematics of the experimental PEMFC test station at IRI (UPCCSIC)

To develop the model, the following modelling assumptions have been considered:

- A mass flow control device ensures a controlled hydrogen supply.

- An auxiliary control system efficiently regulates gas temperatures at five points of the plant (cathode and anode humidifiers, cathode and anode line heaters and stack). 
- A humidity control loop regulates humidifiers moisture to a relative level close to $100 \%$.

- The fuel cell model is one dimensional, so the gases and reactions are considered uniformly distributed in the cells.

- The electrochemical properties are evaluated at the average stack temperature $\left(60^{\circ} \mathrm{C}\right)$, so temperature variations across the stack are neglected.

- The water entering to the cathode and anode is only in vapour phase.

- The effects of liquid water creation are negligible at the gas flow model level.

- The water activity is uniform across the membrane and in equilibrium with the water activity at the cathode and anode catalyst layers.

\section{REDUCED STATE-SPACE MODEL OF A PEM FUEL CELL ANODE LINE}

Previous to designing the observers, a necessary first step involves rearranging the equation presented in [5] and [6] to obtain a reduced order state-space model of the anode subsystem.

For obtaining such model, the procedure consists in coupling the existing differential equations [5], [6] with their auxiliary equations. After this, the system can be represented only in terms of its state variables, external inputs (fuel cell stack current $I_{s t}$ and hydrogen supply $W_{H_{2}}$ ) and measured outputs (anode humidifier pressure $P_{\text {hum }}$ and anode stack pressure $\left.P_{a n}\right)$. Further information about how the overall state space equations were obtained and validated, as well as all their parameters, can be found in [5] and [6].

In what follows, we first review the differential equation describing the evolution of each state and the physical principles that support them [5]. After this, we will describe which variables of the system are available for measurement and also which variables we want to estimate by means of an observer. The meaning and value of all constants and variables appearing in the equations below are described in Appendix.

Let $x_{1}, x_{2}$ and $x_{3}$ denote the air mass inside the anode humidifier, the oxygen mass in the anode channels and the vapor mass in the anode channels, respectively. Denote by $u$ the hydrogen flow of the mass flow regulator $W_{H_{2}}$.

The dynamic of the first state $x_{1}$ is obtained from the mass conservation of hydrogen in the humidifier. The hydrogen flow entering the humidifier is given by $W_{H_{2}}$ and the hydrogen flow leaving this device is $W_{H_{2} \text {,an,in }}$, hence:

$$
\dot{x}_{1}=u-W_{H_{2}, a n, i n},
$$

where $W_{H_{2}, \text { an,in }}\left(x_{1}, x_{2}, x_{3}\right)=C_{0}+C_{1}\left[K_{1} x_{1}-K_{2} \quad\left(R_{h} x_{2}+\right.\right.$ $\left.R_{v} x_{3}\right)$ ].

The dynamic of the second state $x_{2}$ is also derived from the mass conservation principle, but applied to the hydrogen in the anode channels. It relates the input flow to the anode $W_{H_{2} \text {,an,in }}$, its output flow $W_{H_{2} \text {,an,out }}$ and the consumed hydrogen by the electrochemical reaction on the anode's catalyst surface $W_{H_{2} \text {,react }}$ as follows:

$$
\dot{x}_{2}=W_{H_{2}, \text { an }, \text { in }}-W_{H_{2}, \text { an }, \text { out }}-W_{H_{2}, \text { react }},
$$

where

$W_{H_{2}, \text { an }, \text { out }}\left(x_{2}, x_{3}\right)=\frac{K_{a n}\left(P_{a n}-P_{a m b}\right)}{K_{3} \frac{x_{3}}{x_{2}}+1} ; P_{a n}=K_{2}\left(R_{h} x_{2}+R_{v} x_{3}\right)$,

and

$$
W_{H_{2}, \text { react }}\left(I_{s t}\right)=\frac{G_{h} n}{2 F} I_{s t} .
$$

Note that $W_{H_{2} \text {,an,in }}$ is the same hydrogen flow that leaves the humidifier described in (1).

The dynamic of the third state $x_{3}$ models the vapor mass behavior in the anode channels. Given that no water is generated at the anode side, it consists of three flow terms. The first is the water entering the stack $W_{v, a n, i n}$, the second is the water leaving the stack $W_{v, \text { an out }}$. The third term is the water that crosses the membrane from the cathode side $W_{v, \text { mem }}$. Applying the mass conservation principle yields:

$$
\dot{x}_{3}=W_{v, \text { an }, \text { in }}-W_{v, \text { an }, \text { out }}-W_{v, \text { mem }},
$$

where

$$
\begin{gathered}
W_{v, \text { an }, \text { in }}\left(x_{1}, x_{2}, x_{3}\right)=\Phi\left(P_{a n}\right) W_{H_{2}, \text { an }, \text { in }}\left(x_{1}, x_{2}, x_{3}\right), \\
\Phi\left(P_{a n}\right)=\left(\frac{G_{v} P_{\text {sat }, \text { hum }} R H_{\text {hum }}}{G_{h} P_{a n}}+1\right)\left[1-\frac{1}{1-\frac{G_{v} K_{4}}{G_{h}\left(K_{4}-P_{a n}\right)}}\right],
\end{gathered}
$$

and

$$
W_{v, \text { an }, \text { out }}\left(x_{2}, x_{3}, P_{a n}\right)=K_{a n}\left(P_{a n}-P_{a m b}\right)\left[1-\frac{1}{K_{3} \frac{x_{3}}{x_{2}}+1}\right] .
$$

At this point it is important to emphasize that we assume no model for $W_{v, m e m}$, so it is considered as an unknown variable in the model.

\section{A. Measured outputs}

We assume that the following variables are measured:

1) Hydrogen humidifier pressure $y_{1}=K_{1} x_{1}$.

2) Anode pressure $y_{2}=K_{2}\left(R_{h} x_{2}+R_{v} x_{3}\right)$.

3) Hydrogen consumed by the reaction $y_{3}=\frac{G_{h} n}{2 F} I_{s t}$.

Notice that $y_{2}$ is precisely the variable $P_{a n}$ found in the equations (1) and (2) of the system. Moreover, the third output $y_{3}$ is the variable $W_{H_{2} \text {,react }}$ of equation (2).

\section{B. Variables to estimate}

Our aim in this paper is to construct observers to estimate some interesting variables of the system.

The first important variable to estimate is the hydrogen flow $W_{H_{2} \text {,an,in }}$ in (2) that leaves the humidifier and enters the stack anode. This variable cannot be directly measured due its high content of water vapor. Standard mass flow meters cannot be used, because they are highly sensitive to condensation and water droplets.

The second and most important variable to estimate is the membrane water transport $W_{v, \text { mem }}$ appearing in (3). Nafion ${ }^{\circledR}$ based membranes are the most widely used in PEM fuel cells. In these membranes, it is crucial to understand the 
water transport mechanism due to the strong dependency of the proton conductivity and catalyst dynamic performance on the membranes water content and the large amount of swelling due to water uptake. This is not only for analyzing the performance and the durability of fuel cell stacks, but also for developing more reliable systems.

Nevertheless, the mechanism of proton and water transport in Nafion ${ }^{\circledR}$ has not been yet fully understood because of the lack of comprehensive knowledge of its microstructucture and the interaction among the polymer matrix, protons and water molecules which are highly coupled to each other [7]. In this context, $W_{v, \text { mem }}$ is a crucial variable to observe because it takes into account the water flow that crosses the membrane, due to both back diffusion and electrosmotic drag, which are the main inputs to the standard membrane water content models [1]. It is important to stress that because of the unreliable internal model of this term, it is reasonable to consider it as an unknown input to estimate.

\section{OBSERVATION AND IDENTIFICATION}

As announced before, the main goal is to estimate the membrane water transport $W_{v, \text { mem }}$ appearing in equation (3).

The first step towards this goal is estimating the hydrogen

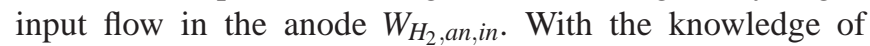
this variable we shall explore two approaches to reach our goal. In the first one, we consider the use of an additional humidity sensor. The inclusion of this sensor allows solving completely the problem without any additional considerations.

In the second approach we consider the problem of estimating the membrane water transport directly from the anode pressure. In such case, it is shown that the problem can not be solved unless additional assumption are made. We consider assuming that the variables are slow enough, so the problem can be recast as a parameter estimation problem.

\section{A. Estimation of the hydrogen input flow in the anode.}

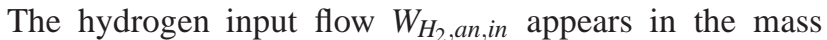
balance equation for the anode (1) as an unknown input. Let us assume that its rate of change is uniformly bounded by a known constant $L_{H_{2}}$ :

$$
\left|\dot{W}_{H_{2}, \text { an,in }}(t)\right| \leq L_{H_{2}}, \quad \forall t \geq 0 .
$$

Let $\hat{W}_{H_{2}, \text { an,in }}$ denote its estimated value. Under the assumption above, it is possible to recover it exactly and in finite-time using the measured output $y_{1}$ and the following Generalized Super-Twisting (GST) observer [8]

$$
\begin{aligned}
\dot{\hat{x}}_{1} & =u-k_{1} \phi_{1}\left(K_{1}^{-1} y_{1}-\hat{x}_{1}\right)-\hat{W}_{H_{2}, \text { an }, i n}, \\
\dot{W}_{H_{2}, \text { an,in }} & =-k_{2} \phi_{2}\left(K_{1}^{-1} y_{1}-\hat{x}_{1}\right),
\end{aligned}
$$

where $k_{1}$ and $k_{2}$ are the gains of the algorithm, and

$$
\begin{aligned}
& \phi_{1}\left(\tilde{x}_{1}\right)=\left|\tilde{x}_{1}\right|^{\frac{1}{2}} \operatorname{sign}\left(\tilde{x}_{1}\right)+\tilde{x}_{1}, \\
& \phi_{2}\left(\tilde{x}_{1}\right)=\frac{1}{2} \operatorname{sign}\left(\tilde{x}_{1}\right)+\frac{3}{2}\left|\tilde{x}_{1}\right|^{\frac{1}{2}} \operatorname{sign}\left(\tilde{x}_{1}\right)+\tilde{x}_{1} .
\end{aligned}
$$

The use of discontinuous injection in (5) allows obtaining an exact estimate despite the fact that $\dot{W}_{H_{2}, \text { an,in }}$ is just uniformly bounded. It is straightforward to verify that the dynamics of the observation errors $\tilde{x}_{1}=x_{1}-\hat{x}_{1}$ and $\tilde{W}_{H_{2}, a n, \text { in }}=W_{H_{2}, a n, \text { in }}-\hat{W}_{H_{2}, \text { an, in }}$ take the same form as in [8]. Then, under the conditions

$$
k_{1}>2 \sqrt{L_{H_{2}}}, \quad k_{2}>2 L_{H_{2}},
$$

the equality $\hat{W}_{\mathrm{H}_{2}, \text { an,in }}=W_{\mathrm{H}_{2} \text {,an,in }}$ will be established in finitetime.

Let us emphasize that observer (4) does not require the value of the humidifier constants $C_{0}, C_{1}$ appearing in the equation that defines $W_{H_{2} \text {,an,in }}$ as a function of the state. The value of these constants change with the operating conditions of the system and need to be experimentally determined [6]. The observer (4) uses only the structure of the system to provide the estimate $\hat{W}_{H_{2} \text {,an, in }}$ that converges to the true value in finite-time.

In what follows, this estimate will be then used to construct an observer for the membrane water transport $W_{v, m e m}$. Figure 2 presents the simulation results for this observer.

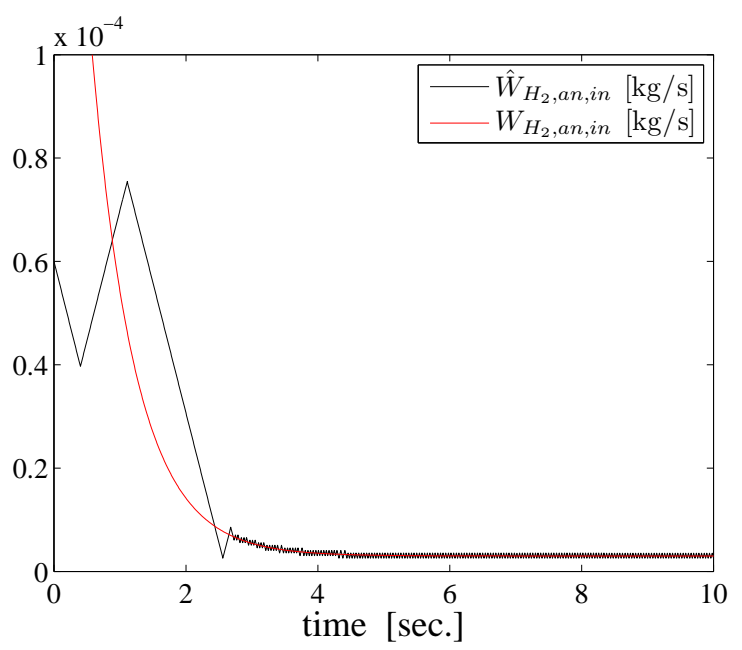

Fig. 2. Simulation results for the estimation of the hydrogen input flow in the anode $W_{H_{2} \text {,an,in }}$ using the GST observer (4) with gains $k_{1}=1 \times$ $10^{-2}, k_{2}=1 \times 10^{-4}$. In red: true value. In black: estimated value.

\section{B. Estimation of the membrane water transport using an humidity sensor.}

Consider the case when the vapor mass in the anode $x_{3}$ can be measured using an humidity sensor at the anode output. Let us assume that such sensor can be modeled by the following linear first-order system

$$
\dot{\xi}=-a \xi+b x_{3}, \quad y_{s}=\xi,
$$

where $y_{s}$ is the output of the sensor. The parameters $a, b>0$ are the (approximate) lag and gain of the sensor, assumed to be known.

In practice, humidity sensors have considerably slow time constants. Moreover, it is difficult to keep them stable when working at high gas relative humidities since their recovery to water condensation is very slow. To circumvent these 
difficulties, it is possible to "predict" the input $x_{3}$ of the sensor by means of a GST observer:

$$
\dot{\hat{\xi}}=-a y_{s}-k_{3} \phi_{1}\left(y_{s}-\hat{\xi}\right)+b \hat{x}_{3}, \quad \dot{\hat{x}}_{3}=-k_{4} \phi_{2}\left(y_{s}-\hat{\xi}\right),
$$

where $k_{3}, k_{4}$ are its gains and the function $\phi_{i}$ are defined as in (5). Under the assumption that $\left|\dot{x}_{3}(t)\right| \leq L_{3}, \forall t \geq 0$, any set of gains satisfying $k_{3}>2 \sqrt{L_{3}}$ and $k_{4}>2 L_{3}$ guarantees that the identity $\hat{x}_{3}(t)=x_{3}(t)$ is kept after finite-time [8].

Using the estimate $\hat{x}_{3}$ constructed above, we can obtain an estimate for $x_{2}$ from $y_{2}$ as follows:

$$
\hat{x}_{2}=\frac{1}{R_{v}}\left(\frac{y_{2}}{K_{2}}-R_{v} \hat{x}_{3}\right),
$$

and also an estimate for the vapor entering and leaving the stack appearing in equation (3):

$$
\hat{W}_{v, \text { an }, \text { in }}=\Phi\left(y_{2}\right) \hat{W}_{H_{2}, \text { an }, \text { in }}, \quad \hat{W}_{v, \text { an }, \text { out }}=W_{v, \text { an }, \text { out }}\left(\hat{x}_{2}, \hat{x}_{3}, y_{2}\right) .
$$

From the properties of observers (4) and (7), the estimates above converge to their corresponding true value after a finite time transient.

Finally, we can construct an estimate $\hat{W}_{v, \text { mem }}$ of the membrane water transport using one more GST observer

$$
\begin{aligned}
\dot{\dot{\bar{x}}_{3}} & =\hat{W}_{v, \text { an }, \text { in }}-\hat{W}_{v, \text { an }, \text { out }}-k_{5} \phi_{1}\left(\hat{x}_{3}-\hat{\bar{x}}_{3}\right)-\hat{W}_{v, \text { mem }}, \\
\dot{\hat{W}}_{v, \text { mem }} & =-k_{6} \phi_{2}\left(\hat{x}_{3}-\hat{\bar{x}}_{3}\right),
\end{aligned}
$$

where, again, $k_{5}, k_{6}$ are its gains and the function $\phi_{i}$ are defined as in (5). If the gains of the observers (4) and (7) are correctly selected as discussed above, and the gain $k_{5}, k_{6}$ satisfy $k_{5}>2 \sqrt{L_{m}}$ and $k_{6}>2 L_{m}$ with $\left|\dot{W}_{v, \text { mem }}(t)\right| \leq L_{m}, \forall t \geq 0$ then the identity $\hat{W}_{v, \text { mem }}(t)=W_{v, \text { mem }}(t)$ will be established and kept after finite-time [8].

\section{Estimation of the membrane water transport from the} anode pressure only.

As discussed in the previous Subsection, humidity sensors come with some intrinsic difficulties in their operation. Hence, real applications motivate the problem of estimating $W_{v, \text { mem }}$ using only the pressure of the anode $y_{2}=K_{2}\left(R_{v} x_{2}+\right.$ $\left.R_{h} x_{3}\right)$.

This problem is more challenging than the previous one, since only a linear combination of the states is measured. For instance, it is impossible to reconstruct exactly $x_{2}$ and $x_{3}$ using the measured outputs described in Section II-A.

To study the solvability of this problem, let us introduce the variable

$$
z=K_{2}\left(R_{h} x_{2}+R_{v} x_{3}\right)-P_{a m b},
$$

where $P_{a m b}$ is the ambient pressure, a constant known parameter defined in (3). Hence, the variable $z$ can be also measured starting from $y_{2}$. Taking one time derivative and using equations (2)-(3) it is possible to write

$$
\dot{z}(t)=U(t)-z(t) \theta_{1}(t)-\theta_{0}(t),
$$

where

$$
U=K_{2}\left[R_{h}\left(W_{H_{2}, \text { an,in }}-W_{H_{2}, \text { react }}\right)+R_{v} W_{v, \text { an }, \text { in }}\right],
$$

and

$$
\theta_{1}=K_{a n} K_{2}\left(R_{v}+\frac{R_{h}-R_{v}}{K_{3} \frac{x_{3}}{x_{2}}+1}\right), \quad \theta_{0}=R_{v} K_{2} W_{v, \text { mem }} .
$$

We have written the explicit dependence on time $t$ in equation (11) to emphasize that $U, \theta_{0}$ and $\theta_{1}$ are all timevarying functions.

The three terms constituting the variable $U$ above can be estimated in finite-time using the measured outputs and the observer (4) as follows. Firstly $W_{H_{2} \text {,react }}$ is directly obtained from the measurement $y_{3}$. Secondly, the flow $W_{H_{2} \text {,an,in }}$ is estimated in finite-time using observer (4). Finally, $W_{v, a n, i n}$ can be estimated in the same way as appears in equation (9), since it depends only on the measured output $y_{2}$ and the estimate $\hat{W}_{H_{2} \text {,an,in }}$ provided also by observer (4). In other words, the variable $U$ can be considered as known. Thus, the problem of estimating the membrane water transport $W_{v, \text { mem }}$ can be restated as estimating the value of $\theta_{0}(t)$ given that $z$ and $U$ are measured.

Since we have only one equation (11) with two unknowns $\left(\theta_{0}, \theta_{1}\right)$, it is not possible to determine their value. Hence, the original problem we formulated can not be solved unless additional assumptions are made.

One useful assumption to make is to consider that $\theta_{0}(t)$ and $\theta_{1}(t)$ change slow enough to treat them as constant parameters in sufficiently small time windows. Under this consideration, the problem is a parameter estimation problem that is known to have a solution if the vector $[z(t), 1]$ is of persistent excitation. Moreover, there exist several wellestablished methods to construct the parameter estimation algorithm.

In what follows we use a modification of the Least Squares Method recently reported in [9]. The modification consist in using Generalized Super-Twisting terms as in (5) to obtain a finite-time estimation of the parameters. Hence, even if the parameters change with time, we can obtain an exact estimate of the parameter in each time window.

For our problem at hand described by (11), the algorithm of [9] takes the following form:

$$
\begin{aligned}
& \dot{\hat{z}}=U+\kappa_{1} \phi_{1}(z-\hat{z})-\left[\begin{array}{ll}
z & 1
\end{array}\right]\left[\begin{array}{l}
\hat{\theta}_{1} \\
\hat{\theta}_{0}
\end{array}\right], \\
& {\left[\begin{array}{c}
\dot{\hat{\theta}}_{1} \\
\dot{\hat{\theta}}_{0}
\end{array}\right]=-\left[\begin{array}{cc}
\kappa_{2} & 0 \\
0 & \kappa_{3}
\end{array}\right] \phi_{2}(z-\hat{z}),}
\end{aligned}
$$

where $\kappa_{i}>0$ are the gains of the algorithm and $\phi_{i}$ are described in (5). The estimate of $\left(\theta_{0}, \theta_{1}\right)$ is given by $\left(\hat{\theta}_{0}, \hat{\theta}_{1}\right)$. It was proved in [9] that if the gains are positive and the regressor $[z(t), 1]$ is of persistent excitation, a finite-time estimation of the parameters is obtained.

\section{Simulation Results}

This section presents two simulation results for the estimation of the membrane water transport from the anode pressure only, as detailed in Section III-C. In the first case, we consider nominal conditions when the outputs described in Section II-A are exactly measured. In the second study, 
measurement noise is added to the measurement of the pressure sensor.

\section{A. Nominal conditions.}

The first simulation results were obtained under nominal operating conditions and without considering noise in the output. When the parameter identifier (12) was turned on, the system was set to operate at a stack current of $1 \mathrm{~A}$. Then at $600 \mathrm{sec}$. a step change in the stack current to $5 \mathrm{~A}$ produces an appreciable change in the variables $\theta_{0}$ and $\theta_{1}$. In Fig. 3, the behavior of the variable $z$ and its reconstructed value $\hat{z}$ using (12) are shown.
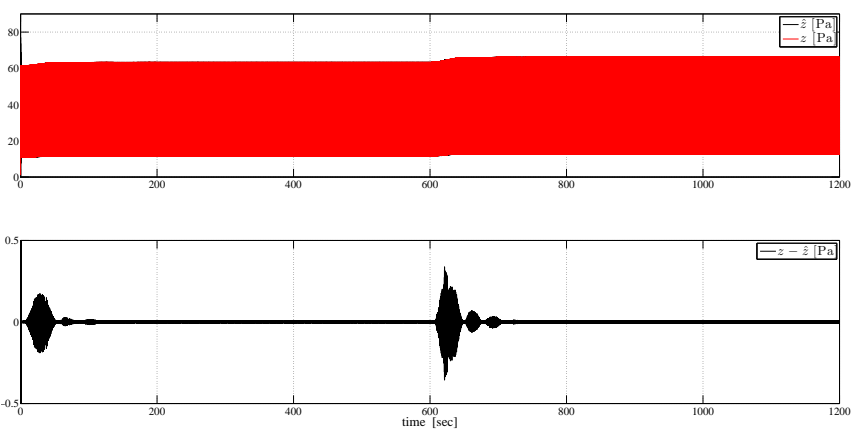

Fig. 3. Variables $z(t)$ vs. $\hat{z}(t)$ obtained in the absence of measurement noises.

Figure 4 presents the the results for the estimation of the parameters. It can be appreciated that the estimation converge exactly and in finite-time.
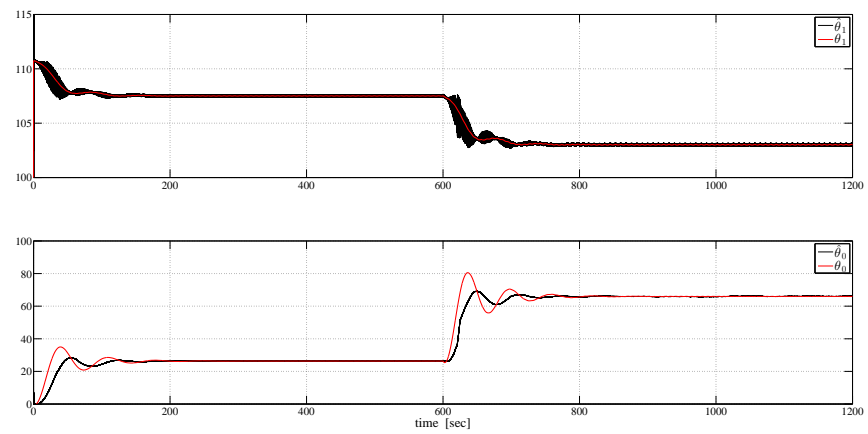

Fig. 4. Estimation of $\theta_{0}$ and $\theta_{1}$ using GSTA based parameter identifier (12) obtained in the absence of measurement noises.

Notice that the exact estimation of $\theta_{0}$ and $\theta_{1}$ depends on the system structure, some standard known parameters and operating conditions. Moreover, reconstruction of $W_{\text {mem }}$ is direct from $\theta_{1}$ and only knowing the vapour specific constant $\left(R_{v}\right)$, the stack temperature $\left(T_{s t}\right)$ and the anode lumped volume $\left(V_{a n}\right)$.

\section{B. Measurement noise in the output $y_{2}(t)$.}

In the second set of simulations, the system was set in the same operating conditions as presented before. However, we add white noise on the variable $z$ representing noise in the pressure sensor. The noise was fixed to have $2 \%$ of the full scale, which is realistic for the current system set-up.

Figures 5 and 6 present the simulation results in this case. Note that despite the presence of heavy noise conditions, the parameter identification algorithm (12) shows good robustness. This result bridges the gap between the simulation stage and the implementation at the laboratory facilities.
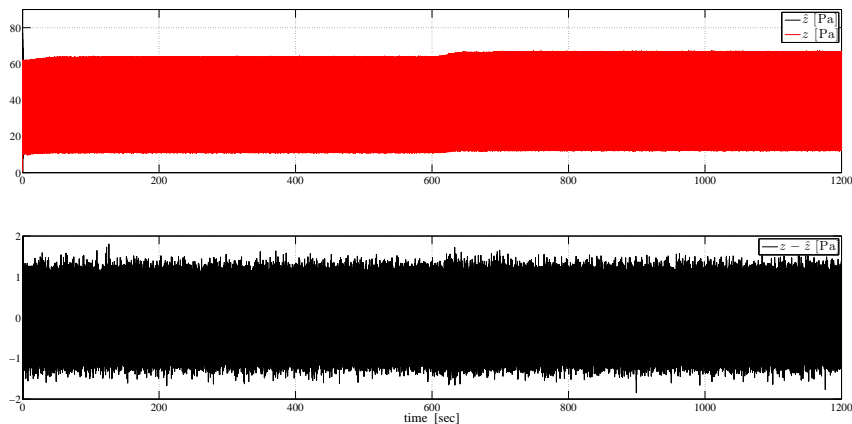

Fig. 5. Variables $z(t)$ vs. $\hat{z}(t)$ obtained in the presence of measurement noises
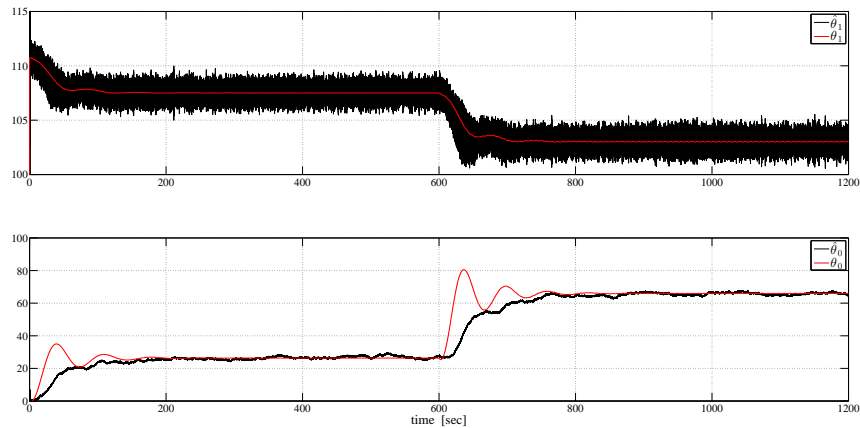

Fig. 6. Estimation of $\theta_{0}$ and $\theta_{1}$ using GSTA based parameter identifier (12) obtained in the presence of measurement noises.

\section{CONCLUSIONS AND FINAL REMARKS}

\section{A. Conclusions.}

We presented an integrated observation and identification approach to estimate the water transport of polymeric membranes in PEM fuel cells. The constructed observers are robust since they depend only on the structure of the system and constants that can be easily determined (e.g., hydrogen or vapor specific constants, anode volume, operating conditions, etc). The only requirement is the use of three sensors in the plant: a pressure sensor in the humidifier, a pressure sensor in the anode and a stack current sensor. From these three measurements, the complete state of the anode line can be recovered as well as $W_{v, \text { mem }}$, which is an important performance variable in PEM fuel cell stacks. Simulation results show the good performance of the presented approach even in presence of noise. 


\section{B. Ongoing work.}

In order to validate the proposed estimation procedure and presented algorithms, the authors are currently working on the experimental phase of this research.

\section{APPENDIX}

The meaning of the all the variables used in this paper are summarized in Table I. Table II presents the specific value of the constants appearing along the text.

\begin{tabular}{|c|c|c|}
\hline Parameter/Variable & Definition & Units \\
\hline$I_{s t}$ & stack/cell current & {$[\mathrm{A}]$} \\
\hline$F$ & Faraday's constant & {$[\mathrm{C} / \mathrm{mol}]$} \\
\hline$G_{h}$ & hydrogen molar mass & {$[\mathrm{kg} / \mathrm{mol}]$} \\
\hline$G_{v}$ & vapour molar mass & {$[\mathrm{kg} / \mathrm{mol}]$} \\
\hline$K_{a n}$ & anode nozzle restriction & {$[\mathrm{kg} / \mathrm{Pa} / \mathrm{s}]$} \\
\hline$n$ & number of cells & [ ] \\
\hline$P_{a m b}$ & ambient pressure & {$[\mathrm{Pa}]$} \\
\hline$P_{a n}$ & stack/cell anode pressure & {$[\mathrm{Pa}]$} \\
\hline$P_{\text {hum }}$ & anode humidifier pressure & {$[\mathrm{Pa}]$} \\
\hline$P_{\text {sat }, \text { hum }}$ & $\begin{array}{l}\text { vapour saturation pressure } \\
\text { in the humidifier }\end{array}$ & [Pa] \\
\hline$P_{\text {sat }, l h}$ & $\begin{array}{l}\text { vapour saturation pressure } \\
\text { in the line heater }\end{array}$ & {$[\mathrm{Pa}]$} \\
\hline$R_{h}$ & hydrogen specific constant & {$[\mathrm{Nm} / \mathrm{kg} / \mathrm{mol}]$} \\
\hline$R_{v}$ & vapour specific constant & {$[\mathrm{Nm} / \mathrm{kg} / \mathrm{mol}]$} \\
\hline$R H_{\text {an,in }}$ & $\begin{array}{l}\text { relative humidity of } \\
\text { the gas entering the anode }\end{array}$ & [] \\
\hline$R H_{\text {hum }}$ & $\begin{array}{l}\text { relative humidity of } \\
\text { the gas leaving the humidifier }\end{array}$ & [ ] \\
\hline$T_{s t}$ & stack/cell temperature & {$[\mathrm{K}]$} \\
\hline$T_{l h}$ & line heater temperature & [K] \\
\hline$V_{a n}$ & anode lumped volume & {$[1]$} \\
\hline$W_{\text {an }, \text { out }}$ & $\begin{array}{l}\text { gas mass flow leaving } \\
\text { the stack/cell anode }\end{array}$ & {$[\mathrm{kg} / \mathrm{s}]$} \\
\hline$W_{H 2}$ & hydrogen supply mass flow & {$[\mathrm{kg} / \mathrm{s}]$} \\
\hline$W_{H 2, a n, \text { in }}$ & $\begin{array}{l}\text { hydrogen mass flow entering } \\
\text { the stack/cell anode }\end{array}$ & {$[\mathrm{kg} / \mathrm{s}]$} \\
\hline$W_{H 2, \text { an,out }}$ & $\begin{array}{l}\text { hydrogen mass flow leaving } \\
\text { the stack/cell anode }\end{array}$ & {$[\mathrm{kg} / \mathrm{s}]$} \\
\hline$W_{H 2, \text { react }}$ & $\begin{array}{c}\text { hydrogen mass flow reacting } \\
\text { at the anode }\end{array}$ & {$[\mathrm{kg} / \mathrm{s}]$} \\
\hline$W_{v, \text { an,in }}$ & $\begin{array}{l}\text { vapour mass flow entering } \\
\text { the stack/cell anode }\end{array}$ & {$[\mathrm{kg} / \mathrm{s}]$} \\
\hline$W_{v, \text { an }, \text { out }}$ & $\begin{array}{l}\text { vapour mass flow leaving } \\
\text { the stack/cell anode }\end{array}$ & {$[\mathrm{kg} / \mathrm{s}]$} \\
\hline$W_{v, \mathrm{mem}}$ & membrane water transport & {$[\mathrm{kg} / \mathrm{s}]$} \\
\hline
\end{tabular}

\section{ACKNOWLEDGEMENTS}

The experimental tests necessary to develop the FC model were performed at the PEM Fuel Cells Laboratory of the Institut de Robòtica i Informàtica Industrial (CSIC-UPC, Barcelona, Spain). The research of C. Kunusch has been supported by the Seventh Framework Programme of the European Community through the Marie Curie actions (GA: PCIG09-GA-2011-293876), Puma-Mind project (GA: FCHJU-2011-1-303419), the CICYT project DPI2011-25649 (MINECO-Spain), the CSIC project MACPERCON (code 201250E027) and the CSIC JAE-DOC Research Programme. J. Moreno gratefully acknowledges the financial support from PAPIIT, UNAM, grant IN111012, and Fondo de Colaboración del II-FI, UNAM, IISGBAS-165-2011.

\begin{tabular}{|c|c|c|}
\hline Parameter & Expression & Value \\
\hline$C_{0}$ & - & $1.0836 \times 10^{-5}$ \\
$C_{1}$ & - & $3.3510 \times 10^{-9}$ \\
$F$ & - & 96485 \\
$G_{h}$ & - & $2.01 \times 10^{-3}$ \\
$G_{v}$ & - & 0.018 \\
$K_{a n}$ & - & $3.2769 \times 10^{-7}$ \\
$K_{1}$ & $T_{s t} R_{h} / V_{a n}$ & - \\
$K_{2}$ & $T_{s t} / V_{a n}$ & - \\
$K_{3}$ & $G_{v} R_{v} / G_{h} / R_{h}$ & - \\
$K_{4}$ & $R H_{a n, i n} / P_{\text {sat }, l h}$ & - \\
$n$ & - & 7 \\
$P_{\text {sat }, \text { hum }}$ & - & $1.388 \times 10^{4}$ \\
$P_{\text {sat, }, \mathrm{h}}$ & - & $1.762 \times 10^{4}$ \\
$R_{h}$ & - & $4.124 \times 10^{3}$ \\
$R_{v}$ & - & 461.5 \\
$R H_{\text {an }, \text { in }}$ & - & 0.74 \\
$R H_{h u m}$ & - & 0.95 \\
$T_{\text {st }}$ & - & 333 \\
$V_{\text {an }}$ & - & 0.02 \\
\hline
\end{tabular}

NUMERICAL VALUES FOR THE CONSTANTS OF THE MODEL

\section{REFERENCES}

[1] McKay, D., Stefanopoulou, A.G., 2004. Parametrization and Validation of a Lumped Parameter Diffusion Model for Fuel Cell Stack Membrane Humidity Estimation. IEEE American Control Conference, Boston, Massachusetts, USA, pp. 816-821, 2004.

[2] Arcak, M., Görgün, H., Pedersen, M., Varigonda, S., 2004. A Nonlinear Observer Design for Fuel Cell Hydrogen Estimation. IEEE Transactions on Control Systems Technology, Vol. 1, 101-110.

[3] Görgün, H., Arcak, M., Barbir, F. 2005. A Voltage-Based Observer Design for Membrane Water Content in PEM Fuel Cells. IEEE AmericanControl Conference, Portland, USA, 4796-4801.

[4] Pukrushpan. J., Stefanopoulou, A., Peng, H., 2004. Control of fuel cell breathing, IEEE Control Systems Magazine, Vol. 24, n. 2, 30-46.

[5] Kunusch, C., Puleston, P., Mayosky, M., Husar, A., 2011. ControlOriented Modelling and Experimental Validation of a PEMFC Generation System. IEEE Transactions on Energy Conversion, Vol. $26 \mathrm{n}$. $3,851-861$.

[6] Kunusch, C., Puleston, P., Mayosky, M., 2012. Sliding-Mode Control of PEM Fuel Cells. Series of Advances in Industrial Control, Springer. ISBN 978-1-4471-2430-6.

[7] Kim, J., 2009. Water Transport in Fuel Cell Membranes Measured by Laser Interferometry. Thesis (Ph.D.), Massachusetts Institute of Technology, Dept. of Mechanical Engineering.

[8] Moreno, J.A., 2011. Lyapunov Approach for Analysis and Design of Second Order Sliding Mode Algorithms. Springer. Chapter 4, 122150.

[9] Moreno, J.A., Guzman, E., 2011. A New Recursive Finite-Time Convergent Parameter Estimation Algorithm. Preprints of the 18th IFAC World Congress Milano (Italy). 